\title{
Survival of a convective core in low-mass solar-like pulsator HD 203608
}

\author{
S. Deheuvels ${ }^{1}$, E. Michel ${ }^{1}$, M. J. Goupil ${ }^{1}$, J. P. Marques ${ }^{1}$, B. Mosser $^{1}$, M. A. Dupret ${ }^{1}$, Y. Lebreton ${ }^{1}$, \\ B. Pichon ${ }^{2}$, and P. Morel$^{2}$ \\ 1 LESIA, CNRS UMR 8109, Observatoire de Paris, Université Paris 6, Université Paris 7, 92195 Meudon Cedex, France \\ e-mail: sebastien.deheuvels@obspm.fr \\ ${ }^{2}$ Laboratoire Cassiopée, CNRS UMR 6202, Observatoire de la Côte d'Azur, BP 4229, 06304 Nice Cedex 4, France
}

Received 6 March 2009 / Accepted 15 January 2010

\begin{abstract}
Context. A 5-night asteroseismic observation of the F8V star HD 203608 was conducted in August 2006 with HARPS, followed by an analysis of the data, and a preliminary modeling of the star (Mosser et al. 2008). The stellar parameters were significantly constrained, but the behavior of one of the seismic indexes (the small spacing $\delta v_{01}$ ) could not be fitted with the observed one, even with the best considered models.

Aims. We study the possibility of improving the agreement between models and observations by changing the physical properties of the inner parts of the star (to which $\delta v_{01}$ is sensitive).

Methods. We show that, in spite of its low mass, it is possible to produce models of HD 203608 with a convective core. No such model was considered in the preliminary modeling. In practice, we obtain these models here by assuming some extra mixing at the edge of the early convective core. We optimized the model parameters using the Levenberg-Marquardt algorithm.

Results. The agreement between the new best model with a convective core and the observations is much better than for the models without. All the observational parameters are fitted within 1- $\sigma$ observational error bars. This is the first observational evidence of a convective core in an old and low-mass star such as HD 203608. In standard models of low-mass stars, the core withdraws shortly after the ZAMS. The survival of the core until the present age of HD 203608 provides very strong constraints on the size of the mixed zone associated to the convective core. Using overshooting as a proxy to model the processes of transport at the edge of the core, we find that to reproduce both global and seismic observations, we must have $\alpha_{\mathrm{OV}}=0.17 \pm 0.03 H_{\mathrm{p}}$ for HD 203608. We revisit the process of the extension of the core lifetime due to overshooting in the particular case of HD 203608.
\end{abstract}

Key words. stars: oscillations - stars: interiors - stars: evolution

\section{Introduction}

For main sequence stars massive enough to show a convective core $\left(M \gtrsim 1.1 M_{\odot}\right.$ for solar-like metallicity), the associated mixed region plays the role of a reservoir for nuclear reactions. The evolution pace of these stars and the time they spend on the main sequence depend directly on the size of this reservoir. The imprecise knowledge we have of the mixing processes, particularly at the boundary of the core, generates large uncertainties on the extension of the mixed core and subsequently on the stellar age and mass for a given set of surface parameters. Among the processes of transport of chemical elements that could contribute to the creation of a mixed zone beyond the edge of the convective core, overshooting is the one invoked most often.

In the deep interior, convective elements rise adiabatically. They are accelerated until they reach the position of convective stability, i.e. $\nabla_{\mathrm{rad}}=\nabla_{\mathrm{ad}}$. Then, the buoyancy forces cause a braking of the eddies in the radiative region. It is, however, unlikely that they should stop abruptly at the boundary between the two regimes. They might penetrate, over a distance $d_{\text {ov }}$, in the regions of stability owing to their inertia, and generate a region of mixing beyond the edge of the core. This phenomenon has been investigated by several authors (see Zahn 1991, and references therein), but no satisfying theoretical or numerical description have been proposed. In practice, this region is modeled as an adiabatic layer above the core, whose thickness is a fraction $\alpha_{\mathrm{OV}}$ of the pressure scale height $H_{\mathrm{p}}\left(d_{\mathrm{ov}}=\alpha_{\mathrm{OV}} H_{\mathrm{p}}\right)$ and where the elements are mixed. While it is admitted that $\alpha_{\text {OV }}$ is only a crude account for the complex processes of mixing at the boundary of the convective core, it is convenient and usual in the modeling of stellar interiors to adopt a representation of these processes depending on this parameter alone. Different studies have led to a wide range of $\alpha_{\mathrm{ov}}$ : between zero (Langer 1986) and about 2 (Xiong 1985). In fact, it is currently admitted that different values of $\alpha_{\text {Ov }}$ might be needed to model stars of different masses and ages (see e.g. Claret 2007). We therefore do not have precise knowledge of the amount of mixing at the edge of the core, and it is one of the main goals of asteroseismology to constrain it with observations (see Lebreton et al. 1995; Michel et al. 2006).

For intermediate-mass and high-mass stars, $d_{\mathrm{ov}}$ is admitted to play an important role (Maeder 1976). The case of low-mass stars is not as clear. When they reach the ZAMS, these stars present a small convective core that disappears almost immediately. It has already been mentioned that an extra mixing at the edge of this early convective core might increase its longevity, by providing first more ${ }^{12} \mathrm{C}$, and then more ${ }^{3} \mathrm{He}$ in the center (Roxburgh 1985). In the specific case of the Sun, core overshooting was added in the models, but it was concluded that it had no relevant impact on the Sun's present structure, unless we add an unreasonable amount of extra mixing. Later on, stellar 
models of low-mass stars suggested that the overshooting at the edge of the core could make it survive almost until the end of the main sequence, although the phenomenon was not explained (Mowlavi 1993).

In this article, we revisit this phenomenon in the case of HD 203608, a low-mass F8V star that presents solar-like oscillations. It was observed with the high-resolution spectrometer HARPS at the ESO 3.6-m telescope in August 2006 (Mosser et al. 2008, hereafter M08). The authors analyzed the oscillation spectrum and identified $15 \ell=0$ and $\ell=1$ eigenmodes. They found a model that agrees with the physical parameters and all the seismic parameters but one: the behavior of the small spacing $\delta v_{01}=v_{n, 0}-\left(v_{n, 1}+v_{n-1,1}\right) / 2$ with frequency. Since HD 203608 is a low-mass star (less than $1 M_{\odot}$ ), the effect of core overshooting was neglected in the preliminary modeling performed in M08. For this range of mass, stars are not expected to have a convective core on the main sequence, except for a small one which disappears shortly after the ZAMS. In Sect. 2, we show that in the case of HD 203608, with a reasonable amount of mixing, the early convective core can survive until the present age. The agreement between observations and models is then greatly improved. In Sect. 3, we explain why the convective core of HD 203608 can survive, even when the burning of ${ }^{3} \mathrm{He}$ is no longer capable of sustaining it.

\section{Modeling of HD 203608}

\subsection{Results from previous modeling of HD 203608}

For their modeling of HD 203608, M08 adopted the following stellar parameters: $T_{\text {eff }}=6070 \pm 100 \mathrm{~K}$ (Jonsell et al. 2005; del Peloso et al. 2005), $L=1.39 \pm 0.13 L_{\odot}$ (from the Hipparcos parallax $\pi=107.98 \pm 0.19$ mas, van Leeuwen 2007) and an observed surface metallicity of $[Z / X]_{\mathrm{f}}=-0.5 \pm 0.1 \mathrm{dex}$, from detailed analysis (see M08). They searched for an optimal model by adjusting the age of the star, its mass, the initial abundance of helium $Y_{\mathrm{i}}$, and the initial metallicity $[\mathrm{Z} / \mathrm{X}]_{\mathrm{i}}$ to fit the following observational constraints: $T_{\mathrm{eff}}, L,[Z / X]_{\mathrm{f}}, \overline{\Delta v}, \delta v_{01}$, and $\delta v_{02}$, where $\overline{\Delta v}$ represents a mean value of the large spacing, and $\delta v_{01}$, $\delta v_{02}$ are the small spacings defined as

$\delta v_{01, n}=v_{n, 0}-\frac{v_{n, 1}+v_{n-1,1}}{2}$

$\delta v_{02, n}=v_{n, 0}-v_{n-1,2}$.

Their best model fits the observational constraints on the star quite well, except for $\delta v_{01}$, for which significant disagreements remain (see Fig. 1).

\subsection{A new modeling including extra mixing at the core boundary}

As shown in Appendix A, the small spacing $\delta v_{01}$ is very sensitive to the central part of the stellar interior. The present disagreement suggests that the way we model these inner regions should be reconsidered. As already mentioned, the models computed in M08 have no convective core. We tried to obtain models of HD 203608 with a convective core here by generating extra mixing at the edge of the core, as suggested by Roxburgh (1985).

We performed a new modeling of HD 203608 including mixing beyond the boundary of the convective core by allowing $d_{\mathrm{ov}}>0$ in our models. We computed stellar models using CESAM2k (Morel 1997), and we derived the mode frequencies from these models with the Liege oscillation code

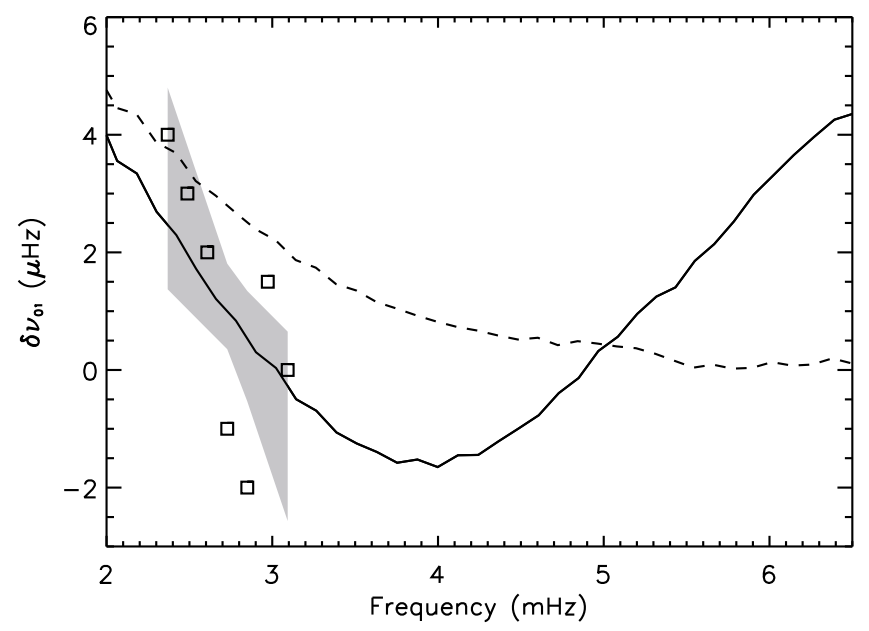

Fig. 1. Variations in the small spacing $\delta v_{01}$ with frequency. The squares represent the observational values of $\delta v_{01}$. The darkened area corresponds to the linear regression of $\delta v_{01}$ derived from the observations within 1- $\sigma$ error bars. The dashed line represents the best model without overshooting (model A), and the full line the best model with overshooting (model B). The frequency axis has been extended to $6.5 \mathrm{mHz}$ in order to exhibit the oscillation of $\delta v_{01}$ for model B.

(LOSC, Scuflaire et al. 2008). We used the same physics as in M08 for our models, apart from the treatment of convection. We preferred to use the more realistic formalism of Canuto \& Mazzitelli (1991). For calibration, we computed a solar model with this treatment of convection and found $\alpha_{\mathrm{CM}}=$ 0.94. As in M08, we adopted this value for our modeling. Overshooting is described, as explained before, as an extension of the adiabatic and mixed region associated to the convective core, over a distance $d_{\text {ov }}$ defined as

$d_{\mathrm{ov}}=\alpha_{\mathrm{OV}} \min \left(r_{\mathrm{c}}, H_{\mathrm{P}}\right)$

where $r_{\mathrm{c}}$ is the radius of the convective core, $H_{\mathrm{P}}$ the pressure scale height, and $\alpha_{\mathrm{OV}}$ the overshooting parameter. The models computed with overshooting showed that the star can still have a convective core at its present age, provided the parameter $\alpha_{\mathrm{OV}}$ is large enough.

\subsection{Optimization}

We looked for an optimal model fitting the global parameters of HD 203608: $T_{\text {eff }}, \log \left(L / L_{\odot}\right)$, and $[Z / X]_{\mathrm{f}}$ (given in Table 1$)$, as well as its seismic parameters. For the seismic constraints, we adopted the mode frequencies obtained in the analysis of M08. We used a mean value of the large separation $\overline{\Delta v}$ and the coefficients of a least-squares linear regression of the small spacings $\delta v_{01}$ and $\delta v_{02}$. The frequency range of the identified modes is indeed small enough to linearize them in the form:

$\delta v_{01} \simeq a_{1}\left(v-v_{0}\right)+b_{1}$

$\delta v_{02} \simeq a_{2}\left(v-v_{0}\right)+b_{2}$,

with $v_{0}$ the frequency of the maximum of the signal $\left(v_{0} \simeq\right.$ $2.75 \mathrm{mHz}$ ). The observational values of these coefficients are given in Table 1 . We thus obtain a set of $N=8$ observational constraints referred to as $p_{i}^{\text {obs }}, i=1, N$. The free parameters are the stellar mass, age, initial helium abundance, metallicity, and the overshooting coefficient $\alpha_{\text {OV }}$ (see Table 1). 
Table 1. Physical and seismic parameters of HD 203608.

\begin{tabular}{|c|c|c|c|}
\hline & Observations & Model A & Model B \\
\hline \multicolumn{4}{|c|}{ Observational constraints } \\
\hline$T_{\text {eff }}(\mathrm{K})$ & $6070 \pm 100$ & 6095 & 6068 \\
\hline$L / L_{\odot}$ & $1.39 \pm 0.13$ & 1.379 & 1.372 \\
\hline$[Z / X]_{\mathrm{f}}(\operatorname{dex})$ & $-0.5 \pm 0.1$ & -0.560 & -0.548 \\
\hline$\overline{\Delta v}(\mu \mathrm{Hz})$ & $120.3 \pm 0.5$ & 120.35 & 120.25 \\
\hline$a_{1}\left(\times 10^{-3}\right)$ & $-5.6 \pm 2.8$ & $-2.4^{\star}$ & -3.6 \\
\hline$b_{1}(\mu \mathrm{Hz})$ & $0.96 \pm 0.67$ & $2.85^{\star}$ & 0.84 \\
\hline$a_{2}\left(\times 10^{-3}\right)$ & $-3.8 \pm 4.2$ & -3.1 & -3.2 \\
\hline$b_{2}(\mu \mathrm{Hz})$ & $6.36 \pm 0.93$ & 6.43 & 5.97 \\
\hline \multicolumn{4}{|c|}{ Free parameters } \\
\hline$\overline{Y_{\mathrm{i}}}$ & - & $0.25 \pm 0.01$ & $0.26 \pm 0.01$ \\
\hline$[Z / X]_{\mathrm{i}}(\operatorname{dex})$ & - & $-0.42 \pm 0.05$ & $-0.50 \pm 0.07$ \\
\hline$M / M_{\odot}$ & - & $0.95 \pm 0.11$ & $0.94 \pm 0.09$ \\
\hline age (Gyr) & - & $6.71 \pm 0.75$ & $6.72 \pm 0.59$ \\
\hline$\alpha_{\mathrm{OV}}$ & - & $\alpha_{\mathrm{OV}}=0^{\sharp}$ & $0.17 \pm 0.03$ \\
\hline$\overline{\chi^{2}}$ & - & $\overline{\overline{9.1}}$ & $\overline{0.8}$ \\
\hline
\end{tabular}

Notes. Derived from the observations, from the best model without overshooting (model A), and from the best model with overshooting (model B). ${ }^{(\star)}$ Quantities not fitted within 1- $\sigma$ error bars; ${ }^{(\sharp)}$ parameter fixed in the optimization.

The best models are obtained by minimizing the $\chi^{2}$ function defined as

$\chi^{2}=\sum_{i=1}^{N}\left(\frac{p_{i}^{\mathrm{obs}}-p_{i}^{\mathrm{mod}}}{\sigma_{i}^{\mathrm{obs}}}\right)^{2}$

where $p_{i}^{\text {mod }}, i=1, N$ are the values obtained in the models for the observationally constrained parameters. In contrast to M08, we used the Levenberg-Marquardt algorithm (as described in Miglio \& Montalbán 2005) to find the minimum of the $\chi^{2}$ function, instead of computing a grid of models. This method is an interpolation between the Newton-Raphson algorithm and the steepest descent method. The steepest descent method is first used, ensuring a rapid approach when the minimum is far. When getting closer to the minimum, the algorithm progressively switches to the Newton-Raphson method for a faster convergence toward the minimum. The advantage is that one can find a minimum with only a few iterations. This kind of optimization is therefore less time-consuming than computing a grid of models with 5 free parameters and a fine grid mesh. The uncertainties on the parameters are obtained from the covariance matrix of the standard errors in the free parameters. We took the best model found in M08 as a first guess and performed two minimizations: one without overshooting, giving best model A, and one with overshooting, giving best model B. Model A is, as expected, very close to the one found by M08. The only difference lies in the error bars, which are bigger here. When using the grid-search method, the error bars are obtained by finding the change in each parameter, which increases $\chi_{\min }^{2}$ by 1 . This approach is only correct if we can neglect the correlation between the different parameters, as explained in Bevington \& Robinson (2003). Several studies have shown that correlations exist between the parameters (Brown et al. 1994; Ozel et al. 2009), which are taken into account in the Levenberg-Marquardt optimization, since we have access to the non-diagonal terms in the covariance matrix. This explains why the uncertainties were underestimated in M08.

Table 1 gives the physical and seismic parameters of both models. For model A, parameters $a_{1}$ and $b_{1}$ are obtained at $1.2 \sigma$ and $2.8 \sigma$ of the observed values, respectively, causing a high value of $\chi_{\min }^{2} \simeq 9.1$ (see Fig. 1). In contrast, for model B, $a_{1}$ and $b_{1}$ are fitted within $0.8 \sigma$ and $0.2 \sigma$, respectively. This results in a significant decrease of $\chi_{\min }^{2} \simeq 0.8$ for model B.

\section{Discussion of the results}

\subsection{Results of the fit}

When considering an extension of the mixed zone associated to the early convective core induced by overshooting, we get a model that fits all the observational constraints better than within $1-\sigma$ of the observed values. This decrease in the $\chi^{2}$ value in fact stems from the survival of the convective core. Indeed, model $\mathrm{B}$ has a convective core that extends over about $3 \%$ of the stellar mass. The withdrawal of this core generates a discontinuity in the chemical composition gradient, hence in the sound speed gradient (see Fig. 2). It has already been established that such a discontinuity induces an oscillation of the mode frequencies as a function of the radial order (see Gough 1990). Provost et al. (1993) derived the expressions of mode frequencies in the case of a discontinuous sound speed profile near the center, in the asymptotic approximation. Using the second-order development they propose, we obtained (see Appendix A) the following expression for $\delta v_{01}$ :

$$
\delta v_{01}(v)=\frac{A}{v}-B \sin \left[2 \pi \frac{n^{\prime}}{\mathcal{P}}+\varphi(v)\right]
$$

where $n^{\prime}, A, B$, and $\varphi(v)$ are defined in Appendix A. With a discontinuous sound speed profile, the small spacing $\delta v_{01}$ oscillates with a period $\mathcal{P}$ corresponding to the ratio between the acoustic radius of the whole star and that of the discontinuity:

$\mathcal{P}=\frac{\int_{0}^{R} \mathrm{~d} r / c}{\int_{0}^{r_{\mathrm{disc}}} \mathrm{d} r / c}$

where $r_{\text {disc }}$ is the radius of the discontinuity. We can see in Fig. 1 that $\delta v_{01}$ indeed oscillates for model $\mathrm{B}$, which was not the case for model A.

When the amount of core overshooting increases, the acoustic radius of the discontinuity in the chemical composition gradient increases and the period of the oscillation decreases (see Eq. (7)). Therefore, the variations in $\delta v_{01}$ become steeper. This shows that the behavior of $\delta v_{01}$ in the models that have a convective core can be adjusted by modifying the extension of the core, e.g. by changing the overshooting coefficient. With an appropriate value of $\alpha_{\mathrm{ov}}$, we can correctly fit the behavior of $\delta v_{01}$ and therefore reduce the $\chi^{2}$ value. We find $\alpha_{\mathrm{OV}}=0.17 \pm 0.03$ for HD 203608. This result provides new input for the studies that seek to establish how convective core overshooting depends on the stellar mass (e.g. Claret 2007).

Stars in a range of mass half way between the presence and the absence of a convective core, such as HD 203608, are interesting objects for studying core overshooting. Indeed, if the seismic analysis of such a star can establish the presence of a convective core, we obtain a firm lower bound for the amount of mixing at the edge of the core. In this case, the extension of the core can be derived from seismic parameters, such as $\delta v_{01}$, and we get a precise estimate of $d_{\mathrm{ov}}$. And if, on the contrary, no convective core is found, a strong upper limit for the extent of the region of extra mixing can be set. 


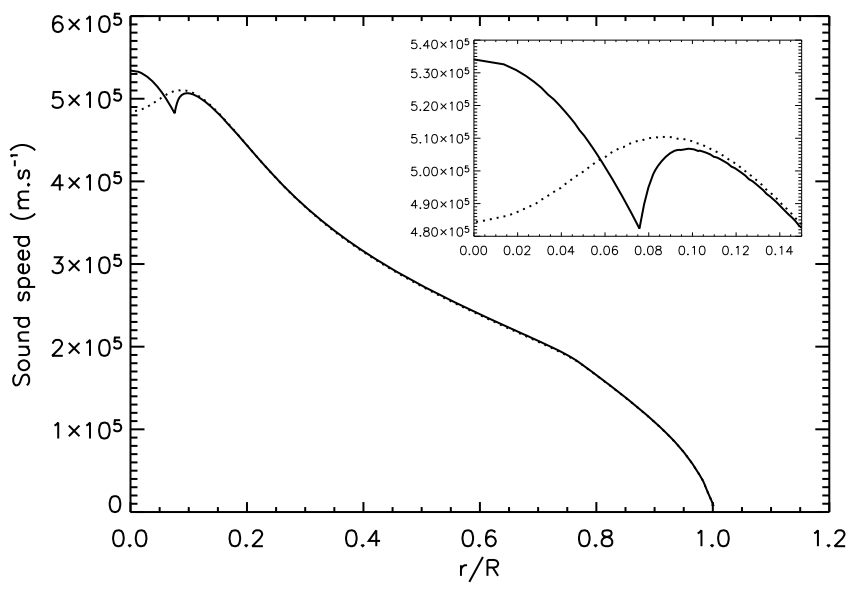

Fig. 2. Sound speed profile for the best model without overshooting (model A, dotted line), and the one with overshooting (model B, full line). A zoom of the curves shows the discontinuity at the edge of the convective core for model B.

\subsection{Survival of the core}

The convective core of HD 203608 disappears at about $200 \mathrm{Myr}$ without overshooting, and survives until about 7 Gyr with $\alpha_{\text {OV }}=$ 0.17 . We explain here this huge difference of longevity caused by overshooting.

\subsubsection{Initial core}

A convective core exists in main sequence models when the energy per unit mass $\varepsilon(m)$ generated by nuclear burning in the center is too high to be transported radiatively. When the nuclear flux is larger than the maximum flux $F_{\text {crit }}$ that can be transported radiatively (corresponding to the radiative flux in the case of critical stability for the Schwarzschild criterion, i.e. $\nabla=\nabla_{\mathrm{ad}}$ ), convective motions transport part of the energy. To ensure the existence of a convective core, we must have high values of the flux of energy $F(m)$ at low $m$, i.e. a high value of $\varepsilon$ in the center. This condition is equivalent to having a steep (negative) gradient $\mathrm{d} \varepsilon / \mathrm{d} m$ in the center. Indeed, the star luminosity is almost entirely produced in the most central parts. If it is generated with a gentle gradient of $\varepsilon$, the value of $\varepsilon$ in the center will be moderate (which is the case for model A, see Fig. 3). Only with a steep gradient of $\varepsilon$ can it reach higher values (see model B in Fig. 3). We therefore use the gradient of $\varepsilon$ hereafter as an indicator of the existence of a convective core.

In most cases, we may assume that the energy production is dominated by a given nuclear reaction chain. Thus, $\varepsilon(m)$ is proportional to $\rho X W T^{v}$, where $\rho$ is the density, $X$ the hydrogen mass fraction, $T$ the temperature, $v$ characterizes the temperature dependence of this specific reaction chain and $\mathrm{W}$ is the mass fraction of the input element corresponding to the reaction (see e.g. Hansen \& Kawaler 1994, chap. 6). We can then write

$\nabla \varepsilon \equiv \frac{\mathrm{d} \ln \varepsilon}{\mathrm{d} \ln m}=\frac{\mathrm{d} \ln \rho}{\mathrm{d} \ln m}+\frac{\mathrm{d} \ln X}{\mathrm{~d} \ln m}+\frac{\mathrm{d} \ln W}{\mathrm{~d} \ln m}+v \frac{\mathrm{d} \ln T}{\mathrm{~d} \ln m}$.

We then see that, to have a steep $\nabla \varepsilon$, the dominant nuclear reactions must have high temperature sensitivity.

For massive and intermediate-mass stars, the gradient of $\varepsilon$ is steep due to the strong temperature dependence of the CNO cycle ( $v \simeq 20$, Hansen \& Kawaler 1994). For HD 203608, when reaching the ZAMS, the temperature in the center is of about $T_{6} \simeq 13$ (where $T_{6}=T / 10^{6} \mathrm{~K}$ ) in our models. At that temperature, the dominant reactions are those of the ppI chain. Since the

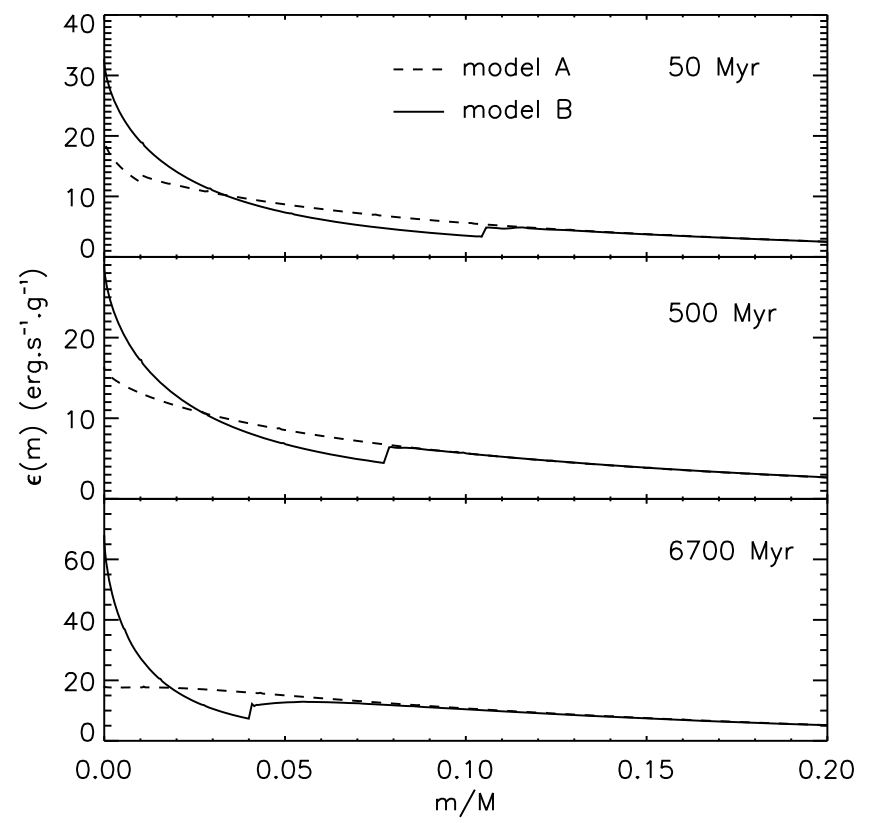

Fig. 3. Profile of the total energy production rate $\varepsilon(m)$ in the center of the star at different stages of its evolution (top: $50 \mathrm{Myr}$, middle: $500 \mathrm{Myr}$, bottom: $6700 \mathrm{Myr}$ ), for model A (dashed line) and B (full line).

pp reaction $-{ }^{1} \mathrm{H}\left(\mathrm{p}, \mathrm{e}^{+} v\right)^{2} \mathrm{H}-$ is the slowest, its generation rate is proportional to that of the whole ppI chain if it has achieved equilibrium. The temperature sensitivity of the ppI chain at equilibrium is therefore the same as that of the pp reaction $v_{\mathrm{pp}}$. Based on Caughlan \& Fowler (1988), we estimate $v_{\mathrm{pp}}=4.1$ at our temperature. This is too weak for the star to have a convective core. However, as pointed out by Roxburgh (1985), the abundances of ${ }^{3} \mathrm{He}$ and ${ }^{12} \mathrm{C}$ are in excess compared to their equilibrium values when the star enters the main sequence. The temperature sensitivities of the burning of ${ }^{3} \mathrm{He}$ to ${ }^{4} \mathrm{He}$ and of ${ }^{12} \mathrm{C}$ to ${ }^{14} \mathrm{~N}$ outside of equilibrium are estimated to be $v_{\mathrm{He} 3}=16.7$ and $v_{\mathrm{C} 12}=18.7$ (Caughlan \& Fowler 1988). We therefore obtain a $\nabla \varepsilon$ about four times steeper than for the equilibrated ppI chain. This ensures that an initial convective core is present.

\subsubsection{Withdrawal of the core}

To achieve equilibrium, the ppI chain requires that the ratio $\left[{ }^{3} \mathrm{He}\right] /[\mathrm{H}]$ corresponds to its equilibrium value. This ratio can be computed by assuming that the deuterium is constantly in equilibrium, which is a good approximation since the destruction of $\mathrm{D}$ is much faster than the other reactions in the chain (see Clayton 1968). It is inversely proportional to the temperature. In the core, the temperature increases towards the center, and the abundance of ${ }^{3} \mathrm{He}$ should decrease with $r$ to achieve equilibrium. Since the elements are mixed in the convective core, the abundance of ${ }^{3} \mathrm{He}$ is constant with $r$, and the reactions are kept outside of equilibrium. The temperature sensitivity therefore remains high, which favors convection. One can say that convection is self-sustained here.

However, $\varepsilon$ also depends on the abundance of the reactant. As the star evolves, the abundance of ${ }^{3} \mathrm{He}$ in the core decreases since it is destroyed faster than it is created. Therefore, $\varepsilon_{\mathrm{He} 3}$ decreases, until the flux of nuclear energy becomes lower than $F_{\text {crit }}$ and the convective core disappears. This happens at an age of about 200 Myr for model A (without overshooting). When the core disappears, the ppI chain quickly achieves equilibrium 


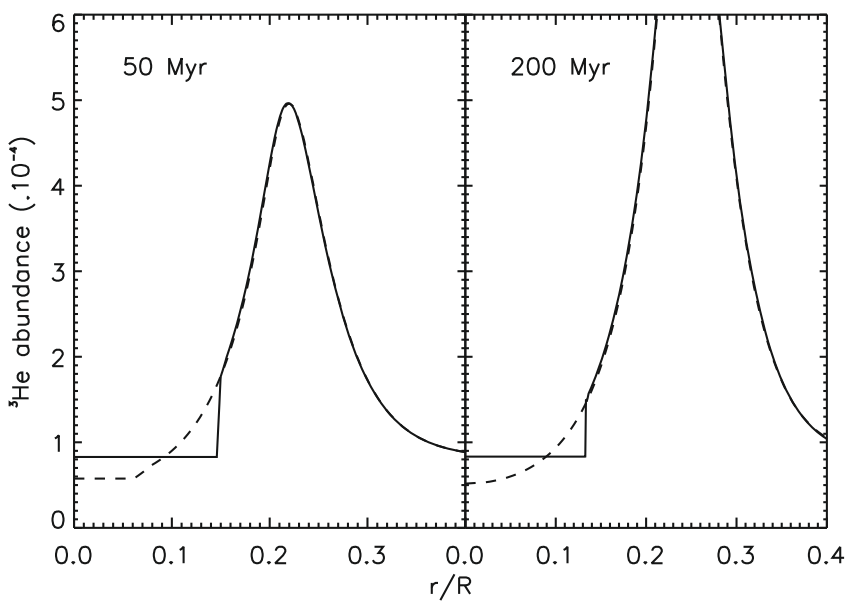

Fig. 4. Abundance of ${ }^{3} \mathrm{He}$ in the center for two ages: $50 \mathrm{Myr}($ left $)$ and $200 \mathrm{Myr}$ (right). The dashed line stands for model A (without overshooting) and the solid line for model B (with overshooting).

(see Fig. 4). The temperature sensitivity becomes that of the ppI chain, which causes $\nabla \varepsilon$ to be less steep. Besides, the elements are no longer mixed in the center, and in Eq. (8), $\mathrm{d} \ln X / \mathrm{d} \ln m>$ 0 and $\mathrm{d} \ln W / \mathrm{d} \ln m>0$. This also contributes to producing a more gentle $\nabla \varepsilon$.

\subsubsection{Effect of an extension of the mixed zone associated to the core}

The existence of a mixed zone at the boundary of the convective core modifies the abundances of elements in the center, and it was already suggested by Roxburgh (1985) that it should increase the lifetime of the core.

The peak we observe in the profile of the ${ }^{3} \mathrm{He}$ abundance (see Fig. 4) is formed when the star reaches the ZAMS. It can be shown that the time required to achieve equilibrium is inversely proportional to the temperature (see Clayton 1968). To the left of the peak and outside the convective core, the ppI chain is already in equilibrium, and the abundance of ${ }^{3} \mathrm{He}$ decreases towards the center because the temperature increases. To the right of the peak, the reactions are not in equilibrium yet, and the abundance of ${ }^{3} \mathrm{He}$ decreases towards the surface since the nuclear reactions get less efficient because of decreasing temperature.

When adding a mixed zone at the edge of the core, we can see in Fig. 4 that the abundance of ${ }^{3} \mathrm{He}$ in the core increases, owing to the peak we just mentioned. Consequently, $\varepsilon_{\mathrm{He} 3}$ increases. The convective core is therefore bigger and survives longer than in the case without additional mixing. If the peak described above did not exist, the mixing would not change the abundance of ${ }^{3} \mathrm{He}$ and would have little effect on the core lifetime. No such peak exists for the ${ }^{12} \mathrm{C}$ profile, and its abundance is small because of the low metallicity of HD 203608. The role of ${ }^{12} \mathrm{C}$ is negligible compared to that of ${ }^{3} \mathrm{He}$ in sustaining the core in the case of HD 203608.

When adding overshooting to our models, we indeed observe an extension of the core's lifetime. However, this extension is quite short. For example, with $\alpha_{\mathrm{ov}}=0.1$, the core disappears at an age of about $1 \mathrm{Gyr}$. In model $\mathrm{B}$, the core is still present at an age of about $7 \mathrm{Gyr}$ with $\alpha_{\mathrm{ov}} \simeq 0.17$. This sudden increase of the core lifetime for $\alpha_{\mathrm{OV}}>0.1$ is in fact caused by the start of the ppII and ppIII reaction chains, and later by the CNO chain, because of the increase in temperature in the center as the star evolves. If the ${ }^{3} \mathrm{He}$ has kept the convective core going until the

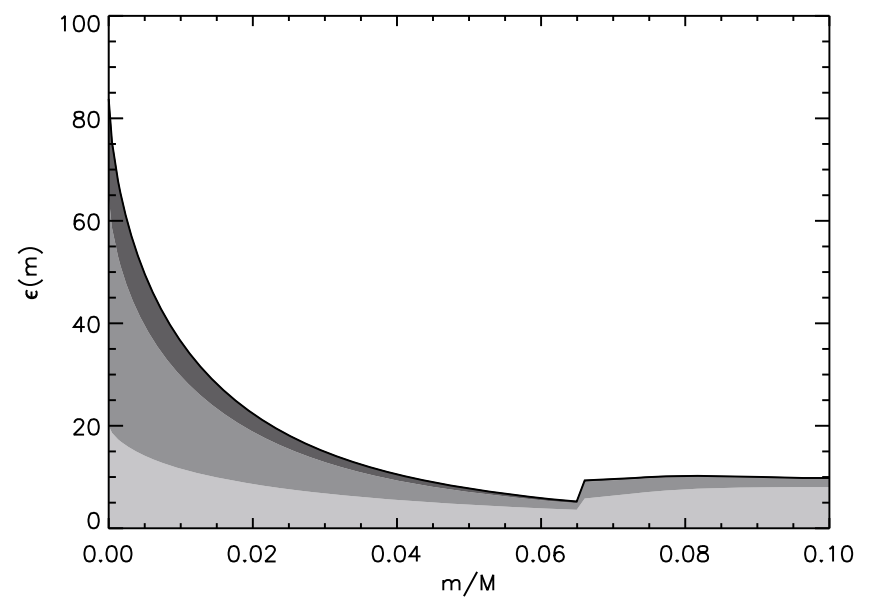

Fig. 5. Energy production rate $\varepsilon(m)$ in the central regions of the optimal model $\mathrm{B}$. The dark line represents the total energy production rate. The grey areas correspond to the luminosity produced by the different nuclear reaction chains (light grey: ppI chain, intermediate grey: ppII chain and dark grey: ppIII chain+CNO cycle).

ppII chain begins to compete, the convection prevents the ppII reactions from achieving equilibrium, in exactly the same way as described above for the ppI chain. The star starts burning ${ }^{7} \mathrm{Li}$ through the reaction ${ }^{7} \mathrm{Li}(\mathrm{p}, \alpha)^{4} \mathrm{He}$ out of equilibrium, with a temperature sensitivity of $v_{\mathrm{Li} 7}=10.8$, which sustains the core. This is what happens for model $\mathrm{B}$. If, on the contrary, the convective core has already disappeared at that time, there is no more mixing in the center and the ppII reactions achieve equilibrium without triggering convection. This is the case for model A.

We can see in Fig. 5 that the ppII chain makes a major contribution to the nuclear production rate $\varepsilon(m)$. The importance of the $\mathrm{CNO}$ reactions is still small. As mentioned above, the energy brought by the ppII chain is almost entirely due to the burning of ${ }^{7} \mathrm{Li}$ outside equilibrium, with the other reactions contributing very little to $\varepsilon_{\mathrm{ppII}}$. If the star were only slightly more evolved, the CNO cycle would take over, causing the convective core to grow.

\subsection{Influence on the evolution}

We notice that, even though the structure of the core is different for our models with and without overshooting, their age is very comparable (see Table 1). This suggests that the survival of the convective core does not significantly influence the global nuclear energy produced by HD 203608 during its evolution. This constitutes a striking difference with higher mass models, where overshooting is known to have a strong impact on the evolution of the star, especially on its age, for a given $T_{\text {eff }}$ and $L$. For high-mass stars, since the temperature dependence of the dominant $\mathrm{CNO}$ cycle is large, it operates in a narrow area in the center, and the wider convective core can act as a reservoir. For HD 203608, the pp chain is dominant, and its temperature dependence is much lower. Therefore, the reactions take place in an area wider than the extent of the convective core, and the mixing has less effect on the evolution.

This means that it would be hopeless to try to characterize an extension of the convective core in this type of star by classical fundamental stellar parameters alone $\left(T_{\mathrm{eff}}, L, M\right)$, as was done by Claret (2007) for higher masses. 


\section{Conclusion}

We present here a modeling of HD 203608 based on the analysis of HARPS data performed in M08. Our main result is that, on this basis, we find strong evidence that this old low-mass star has a convective core. Models with convective cores enabled us to solve the disagreement with observations that was pointed out in M08 for models without convective cores, bringing the $\chi^{2}$ function from 9.1 to 0.8 . All the observed parameters for HD 203608 are now fitted within 1- $\sigma$ error bars.

In the case of our modeling of HD 203608, the value obtained for the $\alpha_{\mathrm{OV}}$ parameter $(0.17 \pm 0.03)$ is strongly constrained. Overshooting was here used as a proxy to model the complex processes of transport at the edge of a convective core, as is usually done in the present state of stellar modeling. Rather than finding a unique absolute value for $\alpha_{\mathrm{OV}}$, the current aim is to try to observationally determine which values of the $\alpha_{\text {OV }}$ parameter are needed to represent stars of different masses and evolution stages. In this respect, the value obtained for HD 203608 constitutes a valuable input for low-mass objects.

We discussed in detail how the existence of a convective core in such an evolved low-mass star can be explained by a reasonable amount of extra mixing (modeled here as core overshooting) inducing the survival of the early convective core. For low-mass stars such as HD 203608, an early convective core exists because of the burning of ${ }^{12} \mathrm{C}$ and ${ }^{3} \mathrm{He}$ outside equilibrium. An extra mixing at the edge of the core increases its lifetime, by bringing more ${ }^{3} \mathrm{He}$ to the center, as mentioned in Roxburgh (1985). Here, we showed that, above a certain amount of overshooting ( $\left.\alpha_{\mathrm{OV}} \sim 0.15\right)$, the burning of ${ }^{3} \mathrm{He}$ out of equilibrium sustains the core until the ppII and ppIII reactions take over. Convection prevents these reactions from achieving equilibrium, and the burning of ${ }^{7} \mathrm{Li}$ outside equilibrium is currently keeping the core convective. This is a transitional phase before the CNO cycle takes over.

The observation of low-mass stars can play a specific role in the study of extra mixing at the edge of stellar cores. Indeed, the presence or absence of a convective core in these stars, which can be established by seismic indicators such as the $\delta v_{01}$ small spacing, strongly constrains the amount of mixing at the edge of the core. This stresses the interest in observing this type of star with ground-based observation campaigns or with the space mission CoRoT (Baglin et al. 2006).

Acknowledgements. We are grateful to A. Noels for useful discussions about the nuclear reactions in the core of low-mass stars.

\section{Appendix A: Expression of the small spacing $\delta v_{01}$ with a discontinuous sound speed profile}

By neglecting the variations in the gravitational potential (Cowling approximation) and using the asymptotic development derived in Tassoul (1980), Provost et al. (1993) obtained the expression of the oscillation frequencies in the case of a discontinuous sound speed profile. The discontinuity is characterized by the parameter $\eta$ :

$\eta=\frac{\rho_{i} c_{i}-\rho_{o} c_{o}}{\rho_{i} c_{i}+\rho_{o} c_{o}}$

where the subscripts $i$ and $o$ describe the quantities at the edge of the convective core in the inner and outer regions. Provost et al. (1993) showed that under these assumptions and provided $\eta$ is small enough, the first-order asymptotic development of the frequencies is given by the expression

$v_{n, \ell}=\left[n^{\prime}+\frac{\ell}{2}-\frac{\eta}{\pi} \sin 2 \pi\left(\frac{n^{\prime}+\ell / 2}{\mathcal{P}}-\frac{\ell}{2}\right)\right] \Delta v$

with

$n^{\prime}=n+\epsilon$

$\Delta v=\left(2 \int_{0}^{R} \frac{\mathrm{d} r}{c}\right)^{-1}$

where $n$ is the radial order of the mode and $\ell$ the degree. The parameter $\epsilon$ depends on the reflective properties of the surface, and its variations with frequency are neglected. When there is a discontinuity, the eigenfrequencies present a sinusoidal oscillatory behavior. These oscillations have an amplitude of $\eta \Delta v / \pi$ and a period $\mathcal{P}$, which corresponds to the ratio of the acoustic radius of the whole star to that of the discontinuity (see Eq. (7)).

Based on this, we can derive the following expression for $\delta v_{01}$ :

$$
\begin{aligned}
\delta v_{01}= & -\frac{\eta \Delta v}{2 \pi}\left[2 \sin \left(2 \pi \frac{n^{\prime}}{\mathcal{P}}\right)+\sin \left(2 \pi \frac{n^{\prime}}{\mathcal{P}}+\frac{\pi}{\mathcal{P}}\right)+\right. \\
& \left.\sin \left(2 \pi \frac{n^{\prime}}{\mathcal{P}}-\frac{\pi}{\mathcal{P}}\right)\right] \\
= & -\frac{2 \eta \Delta v}{\pi} \cos ^{2}\left(\frac{\pi}{2 \mathcal{P}}\right) \sin \left(2 \pi \frac{n^{\prime}}{\mathcal{P}}\right) .
\end{aligned}
$$

As can be seen in Fig. 1, the mean value of the small spacing $\delta v_{01}$ is not zero, as could be expected from Eq. (A.3). To understand this, one needs to push the asymptotic development of the frequencies to the second order. Still from Provost et al. (1993), the expression for the frequencies is given by

$v_{n, \ell}=\left[n^{\prime}+\frac{\ell}{2}+\frac{\ell(\ell+1) V_{1}+V_{2}}{4 \pi^{2} v_{n, \ell}}-\frac{\eta}{\pi} \sin \alpha_{n, \ell}\right] \Delta v$

where

$$
\alpha_{n, \ell}=2 \pi\left(\frac{n^{\prime}+\ell / 2}{\mathcal{P}}-\frac{\ell}{2}-\frac{\ell(\ell+1) V_{3}+V_{4}}{4 \pi^{2} v_{n, \ell}}\right) .
$$

The $V_{j}$ for $j=1,4$ are defined in Provost et al. (1993). They are of the same order of magnitude as $\int_{0}^{R} \mathrm{~d} c / r$. The small spacing $\delta v_{01}$ is therefore sensitive to rapid variations in the sound speed, and particularly in the most central regions, owing to the $1 / r$ factor in the integrand. Using the second-order development of the frequeuncies (Eq. (A.3)), the expression of $\delta v_{01}$ is a bit more complicated. We have

$$
\begin{aligned}
\delta v_{01}= & -\frac{V_{1} \Delta v}{2 \pi^{2} v}-\frac{\eta \Delta v}{2 \pi}\left[2 \sin \left(2 \pi \frac{n^{\prime}}{\mathcal{P}}-\frac{V_{4}}{2 \pi v}\right)\right. \\
+ & \sin \left(2 \pi \frac{n^{\prime}}{\mathcal{P}}+\frac{\pi}{\mathcal{P}}-\frac{2 V_{3}+V_{4}}{2 \pi v}\right) \\
+ & \left.\sin \left(2 \pi \frac{n^{\prime}}{\mathcal{P}}-\frac{\pi}{\mathcal{P}}-\frac{2 V_{3}+V_{4}}{2 \pi v}\right)\right] \\
= & -\frac{V_{1} \Delta v}{2 \pi^{2} v}-\frac{2 \eta \Delta v}{\pi}[ \\
& \cos ^{2}\left(\frac{\pi}{2 \mathcal{P}}\right) \cos \left(\frac{V_{3}}{2 \pi v}\right) \sin \left(2 \pi \frac{n^{\prime}}{\mathcal{P}}-\frac{V_{3}+V_{4}}{2 \pi v}\right) \\
+ & \left.\sin ^{2}\left(\frac{\pi}{2 \mathcal{P}}\right) \sin \left(\frac{V_{3}}{2 \pi v}\right) \cos \left(2 \pi \frac{n^{\prime}}{\mathcal{P}}-\frac{V_{3}+V_{4}}{2 \pi v}\right)\right]
\end{aligned}
$$


This can be rearranged in the form

$\delta v_{01}(v)=\frac{A}{v}+B \sin \left[2 \pi \frac{n^{\prime}}{\mathcal{P}}+\varphi(v)\right]$

where

$$
\begin{aligned}
A= & -\frac{V_{1} \Delta v}{2 \pi^{2}} \\
B= & -\frac{2 \eta \Delta v}{\pi}\left[\cos ^{4}\left(\frac{\pi}{2 \mathcal{P}}\right) \cos ^{2}\left(\frac{V_{3}}{2 \pi v}\right)\right. \\
& \left.+\sin ^{4}\left(\frac{\pi}{2 \mathcal{P}}\right) \sin ^{2}\left(\frac{V_{3}}{2 \pi v}\right)\right]^{\frac{1}{2}} \\
\varphi(v)= & \arctan \left[\tan ^{2}\left(\frac{\pi}{2 \mathcal{P}}\right) \tan \left(\frac{V_{3}}{2 \pi v}\right)\right]-\frac{V_{3}+V_{4}}{2 \pi v} .
\end{aligned}
$$

With a discontinuous sound speed profile, the small spacing $\delta v_{01}$ oscillates with the same period $\mathcal{P}$ as the individual frequencies. In the continuous case, $\eta=0$, and the expression of $\delta v_{01}$ reduces to the usual second-order asymptotic development, which varies like $1 / v$, as is the case for model A (see Fig. 1).

\section{References}

Baglin, A., Auvergne, M., Barge, P. et al. 2006, ESA Special Publication, 1306, 33

Bevington, P. R., \& Robinson, D. K. 2003, Data reduction and error analysis for the physical sciences, ed. P. R. Bevington, \& K. D. Robinson, 3rd edn. (Boston, MA: McGraw-Hill)

Brown, T. M., Christensen-Dalsgaard, J., Weibel-Mihalas, B., \& Gilliland, R. L. 1994, ApJ, 427, 1013
Canuto, V. M., \& Mazzitelli, I. 1991, ApJ, 370, 295

Caughlan, G. R., \& Fowler, W. A. 1988, Atomic Data and Nuclear Data Tables, 40,283

Claret, A. 2007, A\&A, 475, 1019

Clayton, D. D. 1968, (New York: McGraw-Hill), 1968

del Peloso, E. F., Cunha, K., da Silva, L., \& Porto de Mello, G. F. 2005, A\&A, 441,1149

Gough, D. O. 1990, Progress of Seismology of the Sun and Stars, 367, 283

Hansen, C. J., \& Kawaler, S. D. 1994, Stellar Interiors. Physical Principles, Structure, and Evolution, XIII

Jonsell, K., Edvardsson, B., Gustafsson, B. et al. 2005, A\&A, 440, 321

Langer, N. 1986, A\&A, 164, 45

Lebreton, Y., Michel, E., Goupil, M. J., Baglin, A., \& Fernandes, J. 1995, Astronomical and Astrophysical Objectives of Sub-Milliarcsecond Optical Astrometry, 166, 135

van Leeuwen, F. 2007, Astrophysics and Space Science Library, 250

Maeder, A. 1976, A\&A, 47, 389

Michel, E., Baglin, A., Auvergne, M. 2006, ESA Special Publication, 1306, 39

Miglio, A., \& Montalbán, J. 2005, A\&A, 441, 615

Monteiro, M. J. P. F. G., Christensen-Dalsgaard, J., \& Thompson, M. J. 1994, A\&A, 283, 247

Morel, P. 1997, A\&AS, 124, 597

Mosser, B., Deheuvels, S., Michel, E., et al. 2008, A\&A, 488, 635

Mowlavi, N. 1993, IAU Colloq. 137: Inside the Stars, 40, 454

Ozel, N., Dupret, M. A., Michel, E., \& Baglin, A. 2009, A\&A, submitted

Provost, J., Mosser, B., \& Berthomieu, G. 1993, A\&A, 274, 595

Ribas, I., Jordi, C., \& Giménez, Á. 2000, MNRAS, 318, L55

Roxburgh, I. W. 1985, Sol. Phys., 100, 21

Schaller, G., Schaerer, D., Meynet, G., \& Maeder, A. 1992, A\&AS, 96, 269

Scuflaire, R., Montalbán, J., Théado, S., et al. 2008, Ap\&SS, 316, 149

Shaviv, G., \& Salpeter, E. E. 1971, ApJ, 165, 171

Sienkiewicz, R., Bahcall, J. N., \& Paczynski, B. 1990, ApJ, 349, 641

Tassoul, M. 1980, ApJS, 43, 469

Xiong, D. R. 1985, A\&A, 150, 133

Zahn, J.-P. 1991, A\&A, 252, 179 